\title{
Erratum to: Genotyping bacterial and fungal pathogens using sequence variation in the gene for the CCA-adding enzyme
}

Paul Franz, Heike Betat and Mario Mörl

\section{Erratum:}

Unfortunately, the original version of this article [1] contained an error, Fig. 3b was incorrectly labeled.

In regards to Fig. 3b, for the analysis of a possible impact of human DNA in the Vibrio-specific PCR amplification of loop-encoding DNA sequences, the individual lanes with admixture of 0,50 or $100 \mathrm{ng}$ of human DNA contained only DNA of one of the individual Vibrio strains. Hence, the labels 1, 2 and 3 (indicating different Vibrio strains) are erroneous.

A corrected version and legend for Fig. 3 is presented below:

* Correspondence: mario.moerl@uni-leipzig.de

Institute for Biochemistry, Leipzig University, Brüderstr. 34, 04103 Leipzig,

Germany 

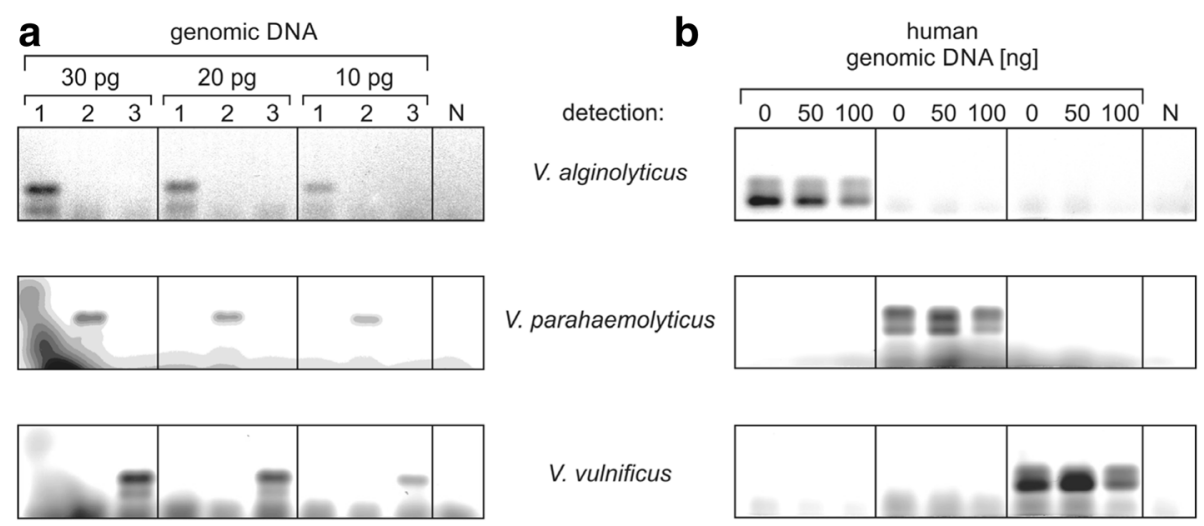

Fig. 3 Multiplex PCR with individual fluorescence-labeled primers for different Vibrio strains. a. Species-specific amplification of the flexible loop-encoding DNA sequence. Indicated amounts of individual genomic DNA (1: V. alginolyticus, 2: V. parahaemolyticus, 3: V. vulnificus) were added to the primer mix. PCR products were visualized in the agarose gel by the different fluorescence of the species-specific primers. Down to $10 \mathrm{pg}$ of each DNA sample were readily detected, without any cross reactivity with the other genomes. $N$, negative control. b. Human DNA does not interfere with the specific detection of Vibrio DNA. $0.1 \mathrm{ng}$ of genomic DNA of the indicated Vibrio strains were mixed with a 500 to 1000 -fold excess (50 and 100 ng) of human genomic DNA in a multiplex PCR and visualized as above. Compared to the positive control (0, no human DNA added), no additional bands appeared, indicating an exclusive and highly specific amplification of Vibrio DNA only. N, negative control with 50 ng of human genomic DNA

Received: 29 July 2016 Accepted: 15 September 2016

Published online: 21 September 2016

\section{Reference}

1. Franz et al. BMC Microbiol (2016) 16:47 doi 10.1186/s1 2866-016-0670-2 